\title{
Correction to: Improved Singleton bound on frequency hopping sequences and optimal constructions
}

\section{Xing Liu $^{1,2}$ (D) Liang Zhou ${ }^{1}$}

Published online: 16 February 2019

๑) Springer Science+Business Media, LLC, part of Springer Nature 2019

\section{Correction to: Des. Codes Cryptogr. https://doi.org/10.1007/s10623-018-0572-4}

The original version of this article contains an error. The presentation of Eq. (14) and an expression in Lemma 4 are incorrect. The correct version is given below.

On Section 2, New bound on FH sequences, the Eq. (14) should be

$$
\begin{aligned}
f\left(\operatorname{gcd}\left(H_{m}+1, N\right)\right)= & \sum_{d \mid \operatorname{gcd}\left(H_{m}+1, N\right)} g(d)=\sum_{d\left|\operatorname{gcd}\left(H_{m}+1, N\right) d^{\prime}\right| d} \mu\left(d^{\prime}\right) f\left(\frac{d}{d^{\prime}}\right) \\
= & \sum_{\substack{d\left|\operatorname{gcd}\left(H_{m}+1, N\right), d^{\prime}\right| d \\
d \neq \operatorname{gcd}\left(H_{m}+1, N\right)}} \mu\left(d^{\prime}\right) f\left(\frac{d}{d^{\prime}}\right) \\
& +\sum_{d^{\prime} \mid \operatorname{gcd}\left(H_{m}+1, N\right)} \mu\left(d^{\prime}\right) f\left(\frac{\operatorname{gcd}\left(H_{m}+1, N\right)}{d^{\prime}}\right) .
\end{aligned}
$$

In other words,

$$
d \mid \operatorname{gcd}\left(H_{m}+1, N\right), \sum_{\substack{\text { sum } \\ d \mid \operatorname{gcd}\left(H_{m}+1, N\right) \\ d 6=\operatorname{gcd}\left(H_{m}+1, N\right)}} d \neq \operatorname{gcd}\left(H_{m}+1, N\right)
$$

The original article can be found online at https://doi.org/10.1007/s10623-018-0572-4.

$凶$ Xing Liu

liuxing4@126.com

Liang Zhou

lzhou@uestc.edu.cn

1 National Key Laboratory of Science and Technology on Communications,

University of Electronic Science and Technology of China, Chengdu 611731, China

2 College of Electrical Engineering and Information Technology, Sichuan University, Chengdu 610065, China 
should be rewritten as

$$
\sum_{\substack{d \mid \operatorname{gcd}\left(H_{m}+1, N\right), d \neq \operatorname{gcd}\left(H_{m}+1, N\right)}}
$$

On proof of Lemma 4, "Let $\bar{n}$ is" should be "Let $\bar{n}$ be".

The original article has been corrected.

Publisher's Note Springer Nature remains neutral with regard to jurisdictional claims in published maps and institutional affiliations. 